\title{
Continual monitoring of intraocular pressure: effect of central venous pressure, respiration, and eye movements on continual recordings of intraocular pressure in the rabbit, dog, and man
}

\author{
R. L. COOPER, D. G. BEAlE, I. J. CONSTABLE, And G. C. GROSE \\ From the Department of Ophthalmology, University Department of Surgery, \\ University of Western Australia
}

SUMMARY A new method has been devised for continual monitoring of intraocular pressure by radiotelemetry. The use of this instrument for monitoring intraocular pressure in a variety of ophthalmic conditions is described.

Diurnal variation in intraocular pressure (IOP) has been recognised for many years. A practical method for noninvasive monitoring of IOP would be important for the diagnosis and management of several forms of glaucoma, particularly the low tension group (Phelps et al., 1974). Intraoperative and postoperative monitoring may be useful for early warning of acute IOP elevation. Such a system will also provide valuable information on the effects of normal physiological processes on intraocular pressure.

Previous attempts at developing such systems have been reviewed elsewhere (Cooper et al., 1979a). In this laboratory we have developed a method of noninvasive passive radiotelemetry of IOP. This communication reports the development of the system up to the present and illustrates its use in conditions designed to simulate clinical situations.

\section{Materials and methods}

Previously described miniature guard ring applanating transensors (AT) (Cooper and Beale, 1977) were mounted in acrylic or Sauflon haptic elements individually designed for the human, rabbit, or dog. The human AT holder was modelled from a standard acrylic haptic whose central optic was removed. The AT was mounted in the lower part of the scleral haptic so that it applanated the inferior

Correspondence to Dr R. L. Cooper, Department of Ophthalmology, University of Western Australia, Royal Perth Hospital, Box X 2213, GPO, Perth, Western Australia 6001.

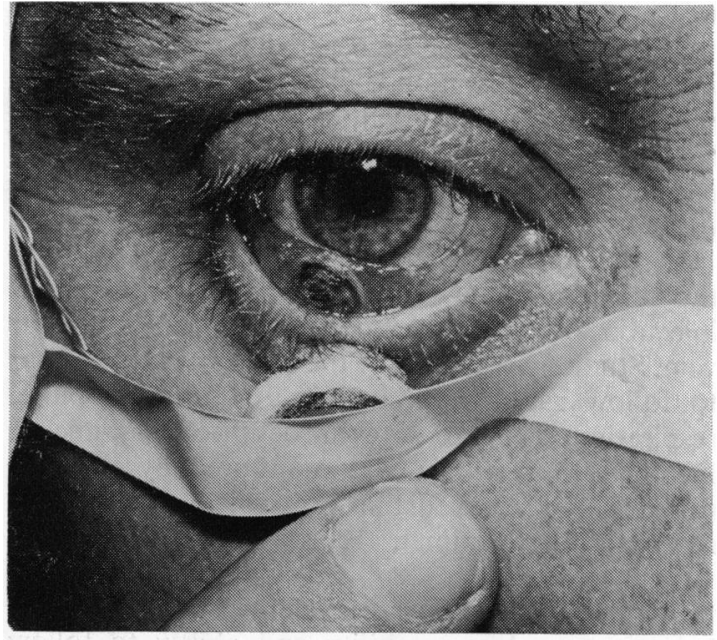

Fig. 1 Continual monitoring of intraocular pressure in man. The lower lid, to which is attached the ACFM aerial, has been everted to show the underlying transensor embedded in an acrylic haptic

sclera under the lower lid. The whole haptic ring was placed in the conjunctival fornix (Fig. 1). The rabbit and dog ATs were mounted in simple bilobed haptics, which rested in the upper conjunctival fornix of the rabbit or were sutured to the sclera in the dog eye.

Intraocular pressure was monitored with an automatic continual frequency monitor (ACFM) invented by one of us (DGB). This miniaturised monitor was fixed to the head by elastic bands. 
The aerial was glued to the surface of the lower lid, overlying the AT, with silicone rubber adhesive (Dow Corning) (Fig. 1). The ACFM induces electromagnetic oscillations in the AT. The frequency of the oscillation is varied continually over the range of the resonant frequencies of the AT. The resonant frequency (Rf) of the AT is directly proportional to IOP. The ACFM monitors these changes in $R f$ and transmits the resonant frequency to a remote radio receiver, whose output is converted to an analogue signal recorded on a standard single channel chart recorder or processed by a Hewlett-Packard $9825 \mathrm{~A}$ computer and subsequently recorded on a 4-channel plotter (HP $9872 \mathrm{~A}$ ). The tracing obtained is thus in the form of a continual variation of $\mathrm{Rf}$, expressed in kilohertz $(\mathrm{kHz})$, which faithfully follows IOP fluctuations.

Recordings of IOP were made in the anaesthetised dog and rabbit and in one conscious person.

\section{GENERAL ANAESTHESIA}

The effects of suxamethonium, the Valsalva manoeuvre, and $\mathrm{PcO}_{2}$ variations on IOP were studied in the anaesthetised dog. The dog was intubated and maintained either under spontaneous respiration or intermittent positive pressure ventilation (IPPV). Central venous pressure (CVP) was recorded in this animal with a Sanborn pressure transducer.

In the rabbits IOP recordings were made with the animals maintained by intermittent intravenous injection of pentobarbitone sodium into an ear vein. Studies were made of the ability of the monitor to follow rapid variations in IOP due to pulse, respiration, and variations in depth of anaesthesia. Depth of anaesthesia was monitored by the lash reflex. The effect of digital pressure on the eye was recorded in this animal.

\section{THE CONSCIOUS HUMAN}

In man transducer stability and ability to follow rapid changes in eye IOP with respiration, eye movements, and lid closure were investigated. The response of the monitor was tested against intermittent recordings of IOP with a Mackay-Marg tonometer (Berkeley Electronics).

\section{Results}

\section{IOP MONITORING UNDER ANAESTHESIA}

The Valsalva manoeuvre was simulated by manual compression of the canine chest during the inhalation stroke of the ventilator. This resulted in a small rise in IOP (Fig. 2). Mean CVP was shown to rise from the initial level of zero to mean pressure of $10 \mathrm{mmHg}$ (Fig. 3).

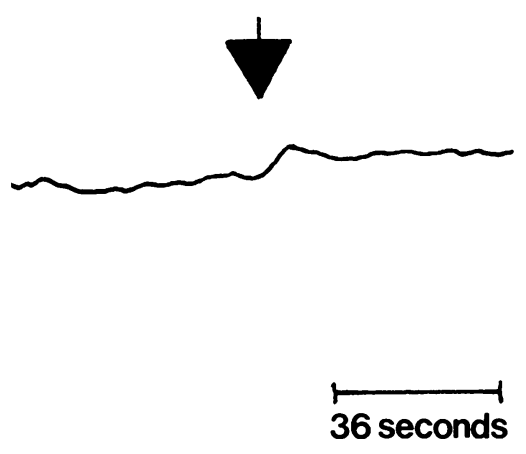

Fig. 2 Continual monitoring of IOP in the dog under anaesthesia. Tracing of IOP record; the arrow indicates the time of compression of the chest in a simulated Valsalva manoeuvre, which resulted in a small rise in IOP

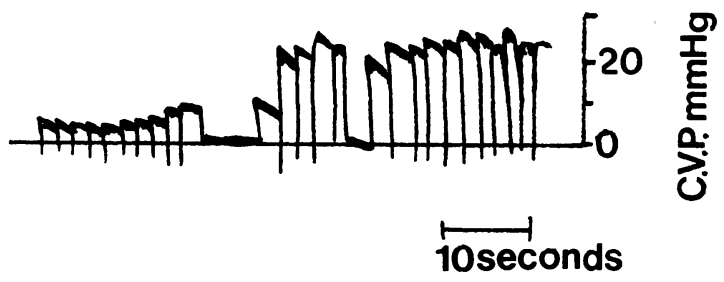

Fig. 3 Trace of central venous pressure recorded by $a$ Sanborn pressure transducer in the anaesthetised dog. The Valsalva manoeuvre was simulated by manual compression of the thorax: mean CVP rose from $0 \mathrm{mmHg}$ to $10 \mathrm{mmHg}$. This recording was made simultaneously with that of Fig. 2

Injection of suxamethonium intravenously in the dog caused a dramatic rise in IOP, which lasted approximately 40 seconds, and settled to a level below baseline. The level to which IOP rose was more than $30 \mathrm{mmHg}$, however, as can be seen from the tracing; the range of the recorder was not high enough to record the maximum peak of IOP (Fig. 4). The CVP recording showed a similar rise in mean venous pressure from zero to approximately $15 \mathrm{mmHg}$, with a gradual decline to a mean CVP of approximately $5 \mathrm{mmHg}$ (Fig. 5).

Variations in $\mathrm{PcO}_{2}$ were accompanied by minimal changes in IOP. Correlation between $\mathbf{P c O}_{2}$ and IOP was poor, whereas correlation between IOP and pH was better.

In the rabbit recordings of IOP showed high frequency excursions of IOP which were synchronous with the pulse. Well-defined low-frequency excursions of IOP due to respiration were observed 


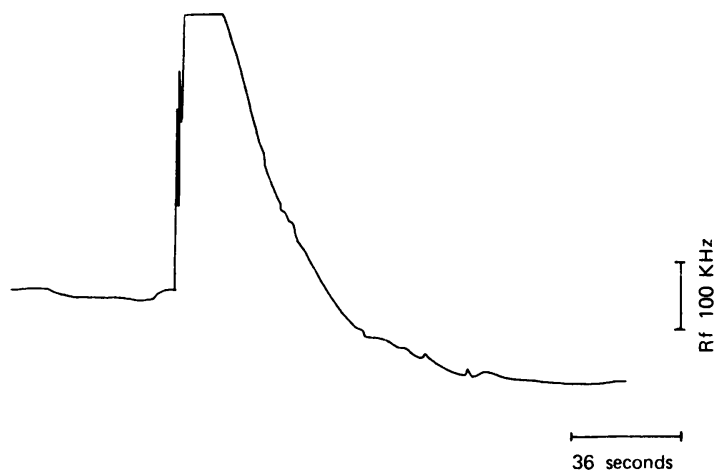

Fig. 4 Continual recording of IOP in the anaesthetised dog by radiotelemetry. The trace shows a sudden rise in IOP due to intravenous suxamethonium injection. IOP falls below baseline as relaxation occurs

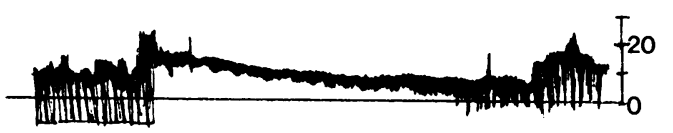

$$
\stackrel{\vdash}{10 \text { seconds }}
$$

Fig. 5 The effect of suxamethonium on central venous pressure recorded by a Sanborn pressure transducer in the anaesthetised dog. Mean CVP rose from $0 \mathrm{mmHg}$ to about $12 \mathrm{mmHg}$. Recording was made simultaneously with that of Fig. 4

in all recordings. An example of such a recording is shown in Fig. 6.

If the depth of anaesthesia in the rabbit was allowed to decrease, there was invariably a rapid rise in intraocular pressure, as the rabbit regained lash reflex, and tone of eyelids and extraocular muscles. An example of a sudden rise in the pressure is shown in Fig. 7. The rapid excursions are due to respiration. With further injection of pentobarbitone and loss of lash reflex the IOP fell to approximately $10 \mathrm{mmHg}$. During the period of lightened anaesthesia the rabbits developed eye rolling movements and eventually blepharospasm, due to the presence of the haptic.

The effect of digital pressure through the eyelids in the rabbit is dramatically shown in Fig. 8. High spikes in intraocular pressure are seen towards the end of the recording, which were due to the application of a Mackay-Marg tonometer in order to correlate the monitor output with applanation pressures. The IOP fell to just below baseline following this pressure on the eye.

\section{MONITORING OF IOP IN THE CONSCIOUS} HUMAN

Recording of the effect of heartbeat and respiration is shown in Fig. 9. As in the rabbit recordings, a high-frequency ripple is superimposed on the main recording, which was synchronous with pulse. The lower frequency variation was due to respiration. The effect of forced inspiration and expiration on IOP is shown in Fig. 10. Inspiration is accompanied by a rapid fall in IOP, with the converse occurring

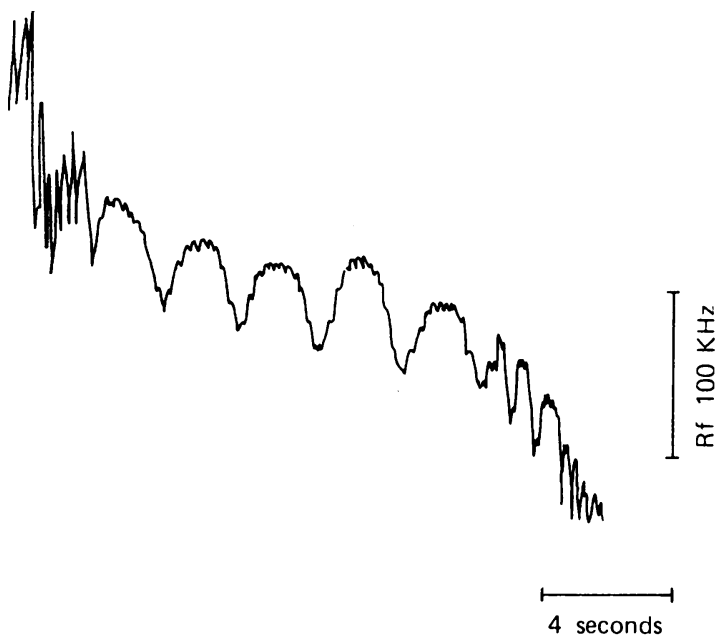

Fig. 6 Continual recording of IOP in the anaesthetised rabbit by radiotelemetry. The ripple is due to pulse and low-frequency variations to respiration

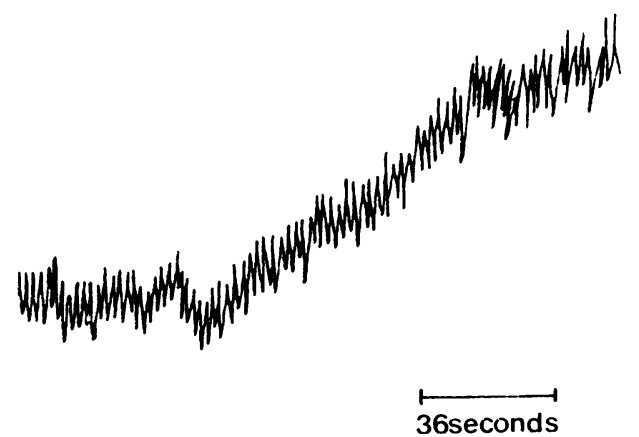

Fig. 7 Response of IOP to lightening of depth of anaesthesia in the rabbit. Blepharospasm and contraction of extraocular muscles cause a rise in IOP lasting about 2 minutes; rapid variations in IOP are due to respiration 


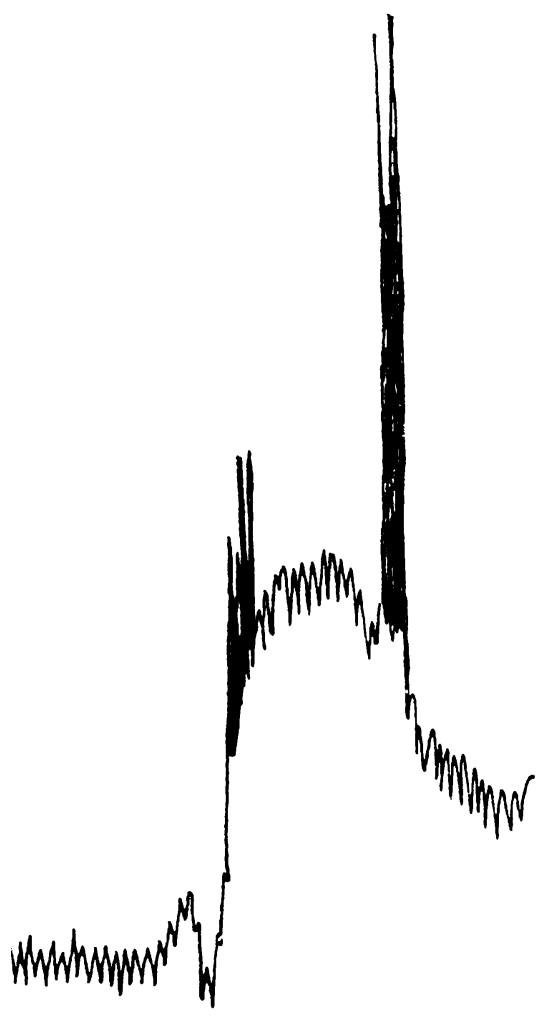

\section{6 seconds}

Fig. 8 Ocular hypertension induced by digital compression of the eye of the anaesthetised rabbit. The high spikes are due to intermittent Mackay-Marg tonometry on expiration. The effect of heartbeat is well shown. Inspiration and breath holding cause an initial reduction in intraocular pressure, followed by a gradual rise as the glottis is closed off (Fig. 11). During this manoeuvre there was always shown to be a short rise in IOP prior to the glottis being closed off and the chest wall relaxing.

Maximal gaze from right to left was accompanied by a rise and fall of IOP. In Fig. 12 the response of<smiles>COCCO</smiles>
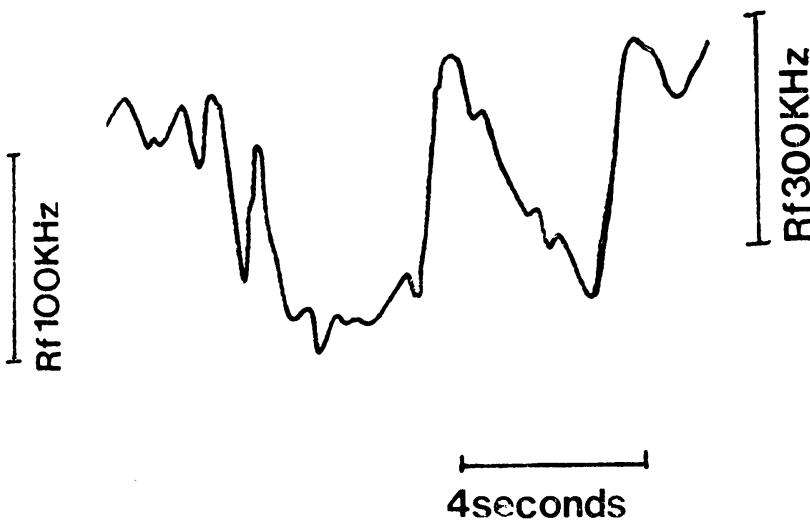

Fig. 10 Continual recording of IOP in the conscious human: the effect of deep breathing on IOP. In $=$ respiration, out $=$ expiration. Deep inspiration (in) decreases resonant frequency by approximately 270 $k H z$. Expiration (out) causes a rise of approximately $300 \mathrm{kHz}$. The rise and fall in IOP cannot be accurately estimated in $\mathrm{mmHg}$ without extensive calibration studies
Fig. 9 Continual recording of IOP by radiotelemetry in the conscious human. Note the excursions due to pulse and respiration
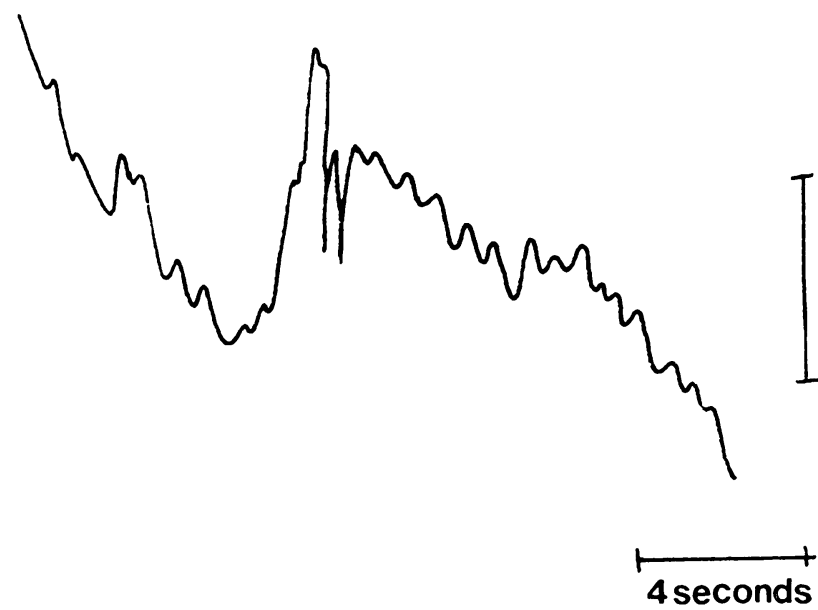


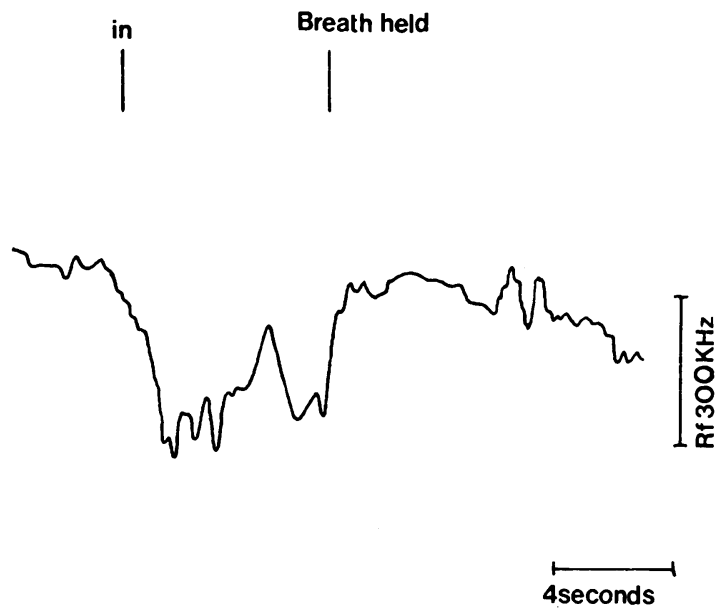

Fig. 11 The effect of breathholding in the conscious human. IOP decreases on inspiration, and increases to baseline on closure of the glottis.

the IOP monitor to right gaze (R) return to the primary position (A) and then full left gaze (L) followed by a repeat of the manoeuvre is shown. These IOP variations are about $10 \mathrm{mmHg}$.

Blepharospasm is thought to cause a rise in intraocular pressure, which was demonstrated in the rabbit experiments. In man closure of the eyelids resulted in a sustained rise in intraocular pressure (Fig. 13). The IOP rose approximately $7 \mathrm{mmHg}$ in this experiment. It will be noted at the beginning of this recording that there are multiple high spikes in the IOP recording. These are blink artefacts, the amplitude of which do not fully reflect IOP, but are due to rapid variations in inductive coupling between the AT and the aerial, as shown by oscilloscopic monitoring.

The response of the monitor to IOP, when tested against intermittent recordings with the MackayMarg tonometer, correlated well with the tonometry readings for up to 2 hours of continual recording. There was then a decline in Rf. We were interested in the effect of continual application of the MackayMarg tonometer to the cornea for purposes of calibration of the instrument for individual eyes. The results of these experiments are reported elsewhere (Cooper et al., 1979b). Fig. 14 illustrates the Mackay-Marg tracing above and the monitor output below. The monitor output faithfully reproduces the rise in IOP induced by the tonometer application. The superimposed fluctuations due to heartbeat, respiration, and tremor of the hand holding the tonometer are reproduced in both tracings.

\section{$\begin{array}{lll}R & A & L\end{array}$}

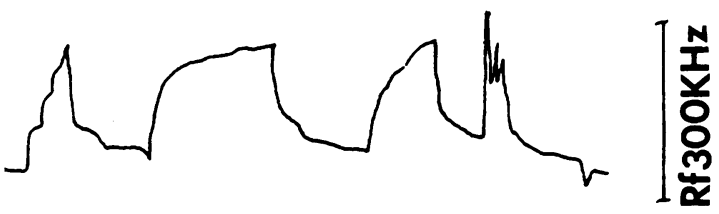

\section{4 seconds}

Fig. 12 The effect of maximal eye movements on IOP in the human: $R=$ right gaze, $A=$ primary position, $L=$ left gaze. Subsequent changes in IOP are due to similar alternating gaze movements ending in a rapid movement to the left, reverting finally to the primary position. True change in IOP cannot be accurately assessed in $\mathrm{mmHg}$. Some of the rise in IOP may be due to distortion of the sclera by the acrylic haptic

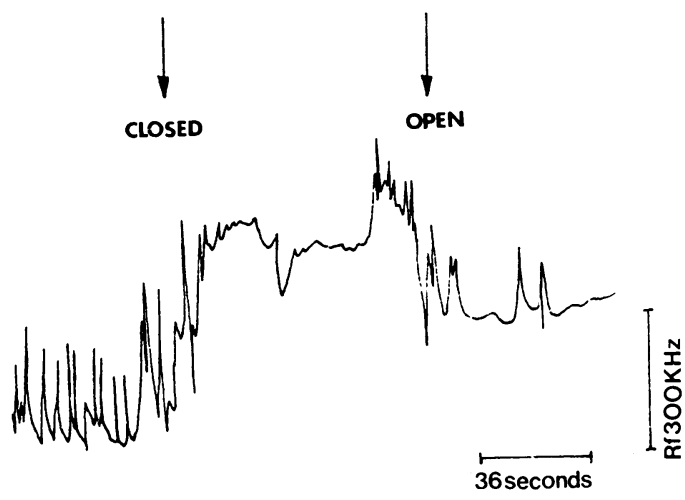

Fig. 13 Continual recording of IOP in the conscious human by radiotelemetry. Closure of the eyelids causes $a$ rise in IOP. The rapid spikes are blink artefacts (see text)

\section{Discussion}

It is well known that IOP is normally maintained at a constant level with only minor diurnal variations. There is some compensation for rapid variations of IOP by the viscoelastic properties of the globe and by eflux of blood from the choroid. However, rapid rises of IOP cannot be fully accommodated by either mechanism. We simulated several conditions that are known to cause acute ocular hypertension during eye surgery. We have demonstrated 2 mechanisms for suxamethonium induced ocular hypertension, namely, contraction 

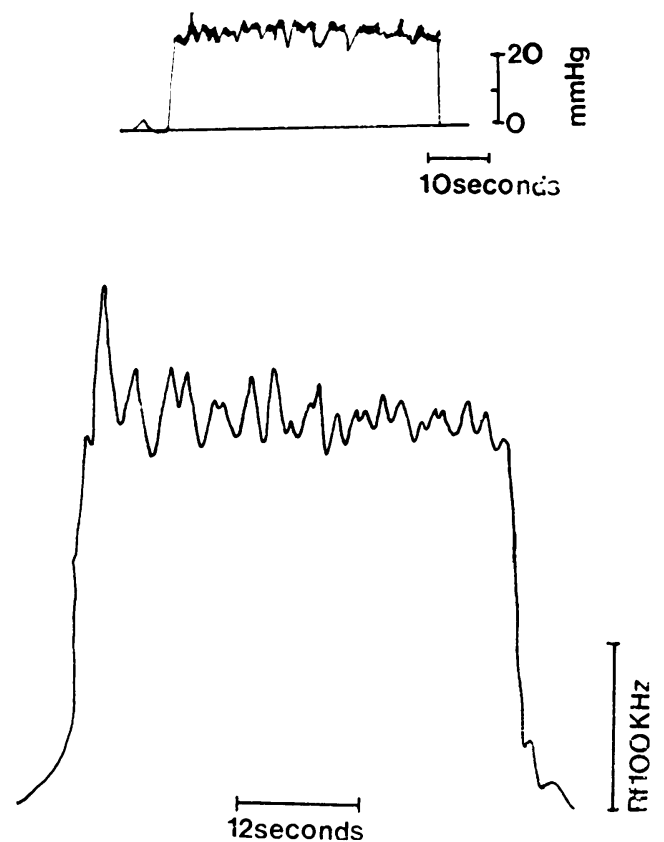

Fig. 14 The effect of continual Mackay-Marg tonometry on IOP in the anaesthetised dog. The upper tracing was obtained from the Mackay-Marg tonometer held on the cornea and the lower tracing from the IOP monitor. The tracings were obtained simultaneously but with different amplitude and time scales. The peaks and troughs recorded by each instrument can be correlated in order to arrive at a calibration of the monitor. These small variations in IOP are due to pulse, respiration, and small movements of the Mackay-Marg tonometer on the cornea. Note the superior sensitivity of the passive telemetric monitor compared to the Mackay-Marg tonometer.

of orbital muscles and a rise in mean CVP. This confirms observations of previous workers (Lincoff et al., 1955; Adams \& Barnett, 1966). We were also able to demonstrate the effect of increasing tone in orbital muscles with lightening of anaesthesia.

In the conscious human we recorded the effects of voluntary eye movements, lid closure, and respiratory excursions. The results thus show that our monitor is capable at least of rapid response and semiquantitative assessment of intraocular pressure over 2 hours.

Despite the capabilities of our system to record noninvasively, several problems remain to be resolved. Individual variations of the scleral rigidity and variation of rigidity with IOP mean that thorough calibration studies will have to be conducted in man with this type of pressure transducer.

The materials from which the AT is constructed have to be improved in order to reduce water vapour permeability for long-term stability. Mechanical problems with the AT and its coupling to the sclera have to be overcome in order to improve accuracy enough to make the instrument capable of quantitative assessment of IOP over at least 24 hours.

The final system, at present being completed, consists of the ACFM reported here, which will transmit IOP information to a portable processing unit, which can sample and record pressure once every 2 minutes for a period of several seconds, over 24 hours. The result of each IOP sample will be recorded on the digital memory board.

Information will be read out from the memory board by the computer and recorded on a disc or plotter. Later a microprocessor and simple chart recorder will be substituted for the computer. The size of the portable processing unit will be such that it can be carried on a waist belt, a shoulder strap, in a handbag, or stored on a bedside table at night. There will be no wired connection between the monitor and the portable processor/memory board. The patient will therefore be unencumbered apart from the requirement to carry the processor around with him or have it within 3 to 6 feet $(2$ to $3 \mathrm{~m}$ ) from the ACFM, which is placed on the head or can be included on a spectacle frame.

This study was supported by funds from National Health and Medical Research Council of Australia, grant no. $77 / 2092$, the Lions Save Sight Foundation of Western Australia, the Royal Perth Hospital Research Foundation. the Ophthalmic Research Institute of Australia, and the Australian Foundation for the Prevention of Blindness.

\section{References}

Adams, A. K., and Barnett, K. C. (1966). Anaesthesia and intra-ocular pressure. Anaesthesia, 21, 202-210.

Cooper, R. L., and Beale, D. (1977). Radiotelemetry of intra-ocular pressure in vitro. Investigative Ophthalmology, 16, 168-171.

Cooper, R. L., Beale, D. G., and Constable, I. J. (1979a). Passive radiotelemetry of intraocular pressure in vivo; calibration and validation of continual scleral guard ring applanation transensors in the dog and rabbit. Investigative Ophthalmologv, in press.

Cooper, R. L., Beale, D. G., Constable, I. J., and Grose, G. (1979b). Passive radiotelemetry of intraocular pressure; investigation into methods for non-invasive calibration in the rabbit and human (submitted for publication).

Lincoff, H. A., Ellis, C. H., De Voe, G., De Beer, E. J., Impastato, D. J., Berg, S., Orkin, L., and Magda, H. (1955). The effect of succinylcholine on intra-ocular pressure. American Journal of Ophthalmology, 40, 501-510.

Phelps, C. D., Woolson, R. F., Koker, A. E. et al. (1974). Diurnal variation in intra-ocular pressure. American Journal of Ophthalmology, 77, 367-377.

ADDED IN PROOF. After submission of this paper for publication a reference was found to direct recording of intraocular pressure in man. It reports similar observations to those observed in man by our monitor (Coleman, DJ, Trokel, S. Arch Ophthalmol 1969; 82: 637-40. 